\title{
Valorising and creating access to innovative medicines in the European Union
}

\author{
Lieven Annemans $^{1}{ }^{*}$, Irina Cleemput ${ }^{2}$, Frank Hulstaert ${ }^{2}$ and Steven Simoens ${ }^{3}$ \\ ${ }^{1}$ Center for Health Economics, Department of Public Health, Ghent University, Ghent, Belgium \\ ${ }^{2}$ Belgian Health Care Knowledge Centre, Brussels, Belgium \\ ${ }^{3}$ Research Centre for Pharmaceutical Care and Pharmaco-economics, Katholieke Universiteit Leuven, Leuven, Belgium
}

Edited by:

Michael P. Jones, Macquarie

University, Australia

Reviewed by:

Nora Ibargoyen, Basque Office for Health Technology Assessment

(Osteba), Department of Health and Consumer Affairs, Spain

Guy Nuyts, Janssen Pharmaceutica, Belgium

\section{*Correspondence:}

Lieven Annemans, Center for Health Economics, Department of Public

Health, Ghent University, UZ-1K3 De

Pintelaan 185, 9000 Ghent, Belgium.

e-mail: lieven.annemans@UGent.be
This Perspective describes (a) the current situation, (b) challenges and initiatives, (c) and formulates recommendations to valorize and create access to innovative medicines in the EU. We are currently still far away from optimal assessment of value for money in the EU. On the one hand, valorizing innovative medicines involves a local appraisal by health technology assessment (HTA) bodies and competent authorities about the value for money, the budget impact, and the local medical need that can be filled with new medicines. Therefore, local priorities and national health care policy environments should be reflected in the processes and criteria used for assessing value for money and ultimately for reimbursement decisions. On the other hand, a pan-European assessment of both relative effectiveness and medical need (including general ethical and social considerations) should be envisaged in order to feed part of the data needed for the local decisions in an efficient way. This could be the task of the European Medicines Agency, HTA bodies, and competent authorities together.

Keywords: pharmaceutical innovation, health technology assessment, cost-effectiveness, relative effectiveness, budget impact, medical need, access

\section{INTRODUCTION}

If investing in health is considered to be an investment of human and financial resources with a view to attain a higher health status for society, it is self-evident that this society should monitor the cost-effectiveness or value for money of this investment. Therefore, once a new medicine can be considered as truly innovative, i.e., showing an added therapeutic value and filling a medical need, the question is whether it is worthwhile to spend public money to cover the cost of this medicine taking into account its therapeutic value, i.e., is the medicine cost-effective?

Explicit decisions must be made about funding a new medicine, mostly by third-party payers (especially social health insurance institutions or national health services or bodies representing them), taking into account a legitimate return on investment for the pharmaceutical company, transparent prices, and value for money. These decision makers are involved with allocating health care budgets and use more and more standardized methods of systematically assessing and appraising such medicines. Criteria such as relative effectiveness, cost-effectiveness, budget impact, medical/therapeutic needs, social, and ethical considerations, play a role in these decisions.

This Perspective describes (a) the current situation of the introduction of innovative medicines in the EU, (b) the challenges and initiatives identified, and (c) formulates recommendations to valorize and create access to them.

\section{CURRENT SITUATION RELATED TO VALUE FOR MONEY ASSESSMENT OF INNOVATIVE MEDICINES}

In deciding whether or not to reimburse the cost of medicinal products, Member States must take into account the principles laid down in Directive 89/105/EEC (European Commission, 1989). The explicit assessment of cost-effectiveness and budgetary impact is however not mandatory at the EU level, but it is the responsibility of the Member States to implement such criteria or not.

Internationally, the first countries implementing such assessments were Australia and Canada in the early 1990s (O'Donnell et al., 2009; International Society for Pharmacoeconomics and Outcomes Research, 2011). The National Institute for Health and Clinical Excellence (NICE) in England and Wales uses a costeffectiveness threshold of $£ 20,000$ per quality-adjusted life year (QALY), although health technologies with an incremental costeffectiveness ratio above this threshold can be recommended for use in the National Health Service if there is a strong case to do so. A review of NICE guidance issued between 1999 and 2005 concluded that health technologies having an incremental costeffectiveness ratio exceeding $£ 30,000$ per QALY were unlikely to be recommended (Raftery, 2006). Judgments about what is regarded as an (un)acceptable ratio are made by NICE's advisory committees, which consist of clinicians and health managers, statisticians, health economists, and patients (Rawlins and Culyer, 2004).

The Belgian Medicine Reimbursement Committee does not use a cost-effectiveness threshold when assessing the value for money of medicines. A retrospective analysis reviewed 824 pharmaceutical reimbursement dossiers that included therapeutic value and that were submitted between 2002 and 2004. Of those dossiers, $81 \%$ gained reimbursement, whereas $19 \%$ did not. If the applicant proved added therapeutic value versus available alternatives, the probability of reimbursement increased to $91 \%$ (Van Wilder and Dupont, 2008). 
In Ireland, new medicines with an ICER below 45,000 $€$ per QALY tended to be reimbursed. If a medicine has an ICER that is significantly higher than other technologies that have been funded or reimbursed, other factors need to be considered, such as: the level of uncertainty associated with the effectiveness and costeffectiveness data, the budget impact and the opportunity cost of investing in the new medicine, the innovative nature of the medicine, and the availability of alternatives (Tilson and Barry, 2010).

The Scottish Medicine Consortium has assessed the value for money of around 600 medicines from 2002 to 2008 (Webb, 2009). Of these 600 medicines, 30\% were accepted for use, 33\% were accepted for restricted use, and 37\% were not recommended. If the cost per QALY was less than or equal to $£ 20,000$ per QALY, there was a probability of $80 \%$ that the medicine was accepted.

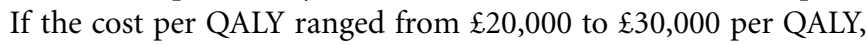
this probability decreased to $42 \%$. A submission was more likely to succeed if one or more of the following conditions was satisfied: innovative treatment, clear effect on relevant clinical outcomes, sizeable QALY benefit and clear cost per QALY case, direct comparison with current practice, transparent modeling with sensitivity analysis, and data relevant to Scottish patients.

A recent review compared the assessments conducted by the NICE in the UK with those of the Australian Pharmaceutical Benefits Advisory Committee (PBAC) and the Canadian Agency for Drugs and Technologies in Health's Common Drug Review (CDR; Clement et al., 2009). According to the authors, the three agencies make recommendations that are consistent with evidence on effectiveness and cost-effectiveness but other factors (medical need, ethical considerations, budget impact) are often important.

It is clear that such conclusions are also valid within the EU, where the context, agency processes, ability to engage in price negotiation, and social values differ among countries.

The role of health technology assessment (HTA) in the process of pricing and reimbursement is becoming crucial, since HTA by definition takes all the required criteria for decision making into consideration and also looks at best practices with technologies. The HTA-methodology might therefore benefit Member States in the implementation of an objective, verifiable, and transparent decision making process. But many challenges remain in place.

\section{CHALLENGES AND INITIATIVES RELATED TO VALUE FOR MONEY ASSESSMENT AND ACCESS TO INNOVATIVE MEDICINES \\ DIFFERENT STRUCTURES AND PROCESSES}

The role of HTA in decision making strongly differs between Member States. Sorenson et al. (2008) explain that divergent structures, processes, and roles may hinder the efficiency of the decision making process and lead to unnecessary duplication of efforts and resource use.

On the structural level, some countries have different institutions involved in HTA, with overlapping responsibilities and tasks and a lack of coordinated recommendations; others have no HTA facilities and are required to rely on foreign evaluations, which may not always come to conclusions that are relevant to their local situation.
On the process level, the applied methodologies are different, limiting the comparability, and transferability across countries. Also, lack of transparency, accountability, and stakeholder involvement is often cited as a common flaw of current systems. Finally, any process should foresee that recommendations need to be reviewed on a regular basis in order to account for the evolving practice in any given disease area.

It is crucial that HTA bodies, national competent authorities, and the European Medicines Agency (EMA) work closer together with transparent assignment of roles and responsibilities in order to avoid duplication of efforts on the one hand or lack of adequate data for decision making on the other hand. The creation of the European Network for Health Technology Assessment (EUnetHTA) has made a significant contribution to the coordination of HTA in the EU. A dialog is currently taking place between HTA agencies and EMA in order to align the agencies' activities and roles with those of the EMA and national competent authorities, as recommended by the High Level Pharmaceutical Forum (European Medicines Agency, 2009).

\section{APPLYING HTA CRITERIA}

Differences in health care systems and in the organization of HTA probably explain a large part of the variance in international HTA. Also, differences in how HTA is perceived, understood or used in various parts of the world may have an important impact on the way it is performed and used. Hence, different applications of HTA may exist even in settings where there are no substantial differences in the health care system or in the organization of HTA.

With regard to the applied criteria, most HTA assessments take a variety of criteria into consideration, including relative effectiveness, cost-effectiveness, budget impact, and medical/therapeutic need. Some countries also explicitly include the public health impact and equity considerations. Some apply a societal perspective, while others apply a more restricted health care perspective. Few countries apply a formal threshold for willingness to pay for one unit of extra health, and even the standard unit of the QALY is challenged by several Member States (Cleemput et al., 2008).

Collaboration on HTA between Member States requires standardization in the structure, transparency, and handling of information in any HTA. Steps toward defining some standards at the international level have been taken by the International Network of Agencies for Health Technology Assessment (INAHTA) and previous European Projects (EUR-ASSESS, ECHTA/ECAHI). More recently, EUnetHTA was established, as a response to the expressed need by the European Commission and Member States for a sustainable European Network for HTA (Kristensen et al., 2009).

The HTA Core Model developed within the EUnetHTA project built on this earlier work (Lampe et al., 2009). The HTA Core Model specifies the questions that should be asked and answered within an HTA and defines and standardizes the structure of an HTA report. To support European collaboration some elements are prioritized over others. They are defined as "core elements." A core element is an assessment element that is considered to be both important for every HTA and transferable to other jurisdictions. A Core HTA is an actual assessment that has been conducted using the HTA Core Model and has considered all core elements 
of all nine HTA domains (i.e., health problem and current use of the technology; description and technical characteristics of technology; safety; clinical effectiveness; costs, economic evaluation; ethical analysis; organizational aspects; social aspects; and legal aspects). Through the wide scope, focus on core elements and the summary chapter, a Core HTA gives an overview of a technology that is likely to be useful in the European context and that can be used as a basis for producing local HTA reports that take into account local circumstances.

\section{UNCERTAINTY AND PERFORMANCE-BASED AGREEMENTS}

A crucial element in value for money assessment is how to deal with uncertainty surrounding effectiveness, costs, and budgetary impact of medicines. A possible way to deal with this uncertainty is the application of risk sharing agreements, or more broadly "performance-based agreements" (Carlson et al., 2009). Performance-based agreements could be defined as formal agreements between a health care payer and a manufacturer where the price level and/or revenue received is related to the (future) performance of the product in order to remain within predefined limits in terms of cost-effectiveness. In these agreements, the eventual effectiveness and costs will drive formal actions oriented to conditional reimbursement, such as companies having to pay for the medicine themselves in case of treatment failure or paying back part of the reimbursed money in case of worse than expected cost-effectiveness. The crucial requirement for performance-based agreements to be effective is that it has to be possible to measure performance and to make reasonably good assessments of the value in "real life." Measuring performance means objectively measuring outcomes on scientifically validated clinical endpoints as well as patient reported outcomes (PROs; Dubois, 2010).

Performance-based agreements are in the interest of the industry, since companies can benefit from new information becoming available on non-clinical positive attributes, such as compliance, impact on quality of life, comfort for health workers, and potential cost-savings. Moreover, predictability of a "fair" list price, reflecting the true value of the product, can be an incentive to further invest in innovative research. Flexible discount and rebate schemes can also provide means to meet the willingness to pay of the health care payer, without touching the global price and the impact on revenue due to benchmarking of pharmaceutical prices across several countries ("external reference pricing"). Additionally, performance-based agreements can be a valuable asset to Compassionate Use and Medical Need Programs to provide access to promising innovative pharmaceutical therapies in domains where an unmet medical need is identified, without compromising the delicate equilibrium between cost and effectiveness, while at the same time enhancing transparency in decision making.

Over the last two decades, numerous performance-based agreements have been put into place. Relative simple "No Cure, no pay" and "Money Back Guarantee"-schemes have been established, such as those for finasteride (US) in benign prostatic hyperplasia, for vardenafil (Denmark) in erectile dysfunction, for valsartan (US and Denmark) in blood pressure treatment, and for bortezomib (UK) in multiple myeloma (National Institute for Health and Clinical Excellence, 2007), alongside with more sophisticated schemes as the UK Risk Sharing Scheme for Multiple Sclerosis (price adjustment to ensure an agreed threshold value for the costeffectiveness ratio; Sudlow and Counsell, 2003), a US “inversed" discount scheme (greater discount in case of greater performance) for sitagliptin (+metformin) in type 2 diabetes and US coverage of disease-related sequelae in case of non-performance for risendronate in the treatment of osteoporosis. However, concerns have been raised about some of these schemes such as the UK Risk Sharing Scheme for Multiple Sclerosis and some have argued that the money should be better allocated to funding a randomized controlled trial of the medicines involved in the scheme (Raftery, 2010).

Today such agreements often take place at the end of a sometimes long negotiation between a pharmaceutical company and a decision maker and are considered as a kind of last resort (European Commission, 2005). Also, early access to new pharmaceutical technologies means early exposure, including the risks involved in using technologies that are not fully evaluated, for instance on long-term safety. This may cause the patient to miss out on optimal treatment for a period of time, or worse, expose patients to a treatment with possible adverse and harmful effects. Other possible disadvantages occur. For establishing sophisticated schemes, data collection and analysis, complex monitoring and review must be feasible and will generate an extra administrative and bureaucratic burden and additional costs for the industry, payers, and administrators. In fact, this might even delay market access. Prices of new medicines might rise in anticipation of subsequent performance-based adjustments. Therapies that prove not to be clinically or cost-effective for the whole target population might be difficult to withdraw if there is proof of benefit for some individual patients. Granting conditional reimbursement could also jeopardize incentives for manufacturers to further invest in additional data collection by means of optimal evaluative study design and study conduct. Furthermore, the clinical community may regard this experimental research to be unethical and patients could be reluctant to participate in such schemes.

\section{AFFORDABILITY, ACCESS, AND SOLIDARITY}

Innovative medicines are generally launched in markets where the companies can get a high price for them and then launched in other markets later. These later markets decide the price by looking at prices in other countries, so that there is little price difference, which makes it difficult for low-income countries to have affordable access to medicines (Docteur and Paris, 2008). A crucial challenge in the EU context is to make valuable innovative medicines accessible to all EU citizens, which requires solidarity within Member States and solidarity between Member States. Ideally, valuable innovative medicines should be launched in all markets and available there at an affordable price.

With regard to within country solidarity, it is important to understand that cost-effectiveness estimates do not account for distributive aspects (poor/rich; young/old; rare diseases, ...). The utilitarian vision on health investment goes against the principle that everyone has the right to the same quality of health care. Perhaps the societal willingness to pay for QALYs or the QALYs themselves should be weighted for factors such as severity of disease. Reflection is needed on a country level about the elements 
based on which such solidarity and weighing can be achieved, and all stakeholders need to be involved. A good example is given by the citizen's council reports in the UK (National Institute for Health and Clinical Excellence, 2005).

But reflection is also needed at the EU level, related to between Member State solidarity. In principle all Europeans have the right to the same quality of health care and it is not acceptable that in case of a severe condition inhabitants of country $\mathrm{X}$ have access to new treatment whereas inhabitants of country Y do not. Obviously, due to differences in health systems and priorities, a solution for this problem will be very difficult to achieve.

It should perhaps be explored whether a system of price differentiation can be applied in function of countries' GDP per capita in order to enable such access. Although these kind of pricing systems reflect in a sense the principles of solidarity and equity, as countries with more resources would carry part of the financial burden of more vulnerable countries, reflection on implementation is needed. The establishment of a systematic price differentiation scheme would call for the determination of some sort of European "base" price, reflecting the value and the value for money of medicines on a European level. A European "average" price, reflecting a European "average" value for money would not necessarily reflect the true value for money for individual countries given that health priorities can differ between vulnerable countries and countries with more financial power. It remains to be examined if adjustments or price differentiation, merely on GDP, will suffice, and should they not, which other criteria could be considered. At the same time, as these schemes could in a way be considered as external reference pricing schemes, they could limit the scope for individual countries to negotiate (for instance compensation mechanisms or performance-based agreements) on prices of the concerned pharmaceuticals.

It should also be examined how these differential pricing systems can be structured in a way that is compatible with the European regulation on the internal market, more specifically on free traffic of goods, the freedom to provide services, and the freedom of establishment. Free parallel import and export for instance, should not be hampered or hinder itself the efficiency and sustainability of such structures. As a minimum, systems should be put in place that guarantee that those medicines offered on the local market effectively reach local patients. A way forward could be to consider innovative pharmaceutical medicines as a social insurance service, hence not requiring the rules of the EU internal market.

\section{ADDITIONAL CHALLENGES FOR ORPHAN MEDICINES}

For orphan medicines, additional challenges occur. Indeed, given their high price for an often modest health benefit, orphan medicines are often unlikely to be cost-effective, at least if the costeffectiveness of an intervention is judged based on its cost per QALY gained, and this cost per QALY is compared to a fixed threshold value.

Possibly, additional criteria that are not included in the traditional cost per QALY measure (e.g., the seriousness of the health condition; the availability of other therapies to treat the disease; and the cost to the patient if the medicine is not reimbursed, ...) can even be more relevant to inform decisions on orphan medicines. The question arises as to how these various considerations can be aggregated. In contrast with medicines for nonorphan diseases, how can the often poor cost-effectiveness ratio, weak clinical data, small health benefit, high cost, and absence of an alternative therapy for orphan medicines be taken into account in a payer's decision to cover such a medicine? It could be argued that the cost-effectiveness threshold value should be higher for medicines to which society attaches a high social value. Orphan medicines may attract a high social value, although future research will have to elicit social values ascribed to various health technologies.

The newly agreed EU principle that "Member States, stakeholders, and the Commission should strengthen their efforts to ensure access to orphan medicines in all EU Member States," led to the EU exchange of knowledge on the scientific assessment of the clinical added value of orphan medicines, specific pricing and reimbursement mechanisms and an early dialog on research and development, as well as an increased awareness on rare/orphan diseases (European Commission, 2005). Still it is felt that EU policy makers should share more intensively their considerations and criteria when deciding on adopting (reimbursing) orphan medicines and more efforts should be made to coordinate processes and criteria.

\section{ADDITIONAL CHALLENGES FOR PERSONALIZED MEDICINE}

A special challenge exists in the assessment of medicinal products whose use is or can be dependent on the result of genetic tests. Often the decision makers dealing with the value for money of such tests are different from those dealing with medicines, and the criteria applied are different as well, leading to additional problems of lack of coordination. The same principles of cost-effective use and budget impact should also apply to such genetic tests, and the decision making for tests and associated medicines must be aligned.

\section{RECOMMENDED WAYS FORWARD}

We are currently still far away from optimal assessment of value for money in the EU. On the one hand, valorizing innovative medicines involves a local appraisal of the value for money, the budget impact, and the local medical need that can be filled with new medicines. Although unmet medical needs tend to be the same across the EU, medical needs may differ between countries when for example a medicine for a rare disease is reimbursed in one country, but not in another country. Indeed, local priorities and national health care policy environments should be reflected in the processes and criteria used for assessing value for money and ultimately for reimbursement decisions. On the other hand, a pan-European, coordinated or even integrated assessment of both relative effectiveness and medical need (including general ethical and social considerations) should be envisaged in order to feed part of the data needed for the local decisions in an efficient way. Our proposal for a Joint Initiative for Medicines could be the task of EMA, HTA bodies, and national competent authorities together.

Local assessment of medical need, supplementary ethical and social aspects, cost-effectiveness, and budget impact should then remain the responsibility of the Member States, although 
Table 1 | Assessment of innovative medicines in the European Union.

Assessment criteria for innovative medicines
Current

\begin{tabular}{ll}
\hline Centralized & Local \\
EMA & HTA and competent \\
& bodies
\end{tabular}

Future

\begin{tabular}{ll}
\hline Integrated & Local with exchange \\
Joint initiative & HTA bodies and \\
for medicines & competent bodies
\end{tabular}

\begin{tabular}{|c|c|c|c|c|}
\hline Efficacy & $\checkmark$ & $\checkmark$ & $\checkmark$ & \\
\hline Safety & $\checkmark$ & $\checkmark$ & $\checkmark$ & \\
\hline Relative efficacy & $\checkmark$ & $\checkmark$ & $\checkmark$ & \\
\hline Relative effectiveness & & $\checkmark$ & $\checkmark$ & \\
\hline EU medical need & & & $\checkmark$ & $\checkmark$ \\
\hline Local medical need & & $\checkmark$ & $\checkmark$ & $\checkmark$ \\
\hline Ethical and social aspects & & $\checkmark$ & $\checkmark$ & $\checkmark$ \\
\hline Organizational aspects & & $\checkmark$ & & $\checkmark$ \\
\hline
\end{tabular}

The Joint Initiative for Medicines refers to our proposal for a joint initiative between EMA, HTA bodies, and national competent authorities.

cross-border exchange of methods, information, and decisions would be highly valuable. Note that local assessments may be based on core international cost-effectiveness and budget impact models that are adapted to the local setting.

According to this scheme, similar outcomes of relative effectiveness assessments will nevertheless allow different Member States to come to different reimbursement decisions based on either differences in value judgment or budgetary evaluations, or on any

\section{REFERENCES}

Carlson, J. J., Garrison, L. P., and Sullivan, S. D. (2009). Paying for outcomes: innovative coverage and reimbursement schemes for pharmaceuticals. J. Manag. Care Pharm. 15, 683-687.

Cleemput, I., Neyt, M., Thiry, N., De Laet, C., and Leys, M. (2008). Threshold Values for Cost-Effectiveness in Health Care. Brussels: Belgian Health Care Knowledge Centre.

Clement, F. M., Harris, A., Li, J. J., Yong, K., Lee, K. M., and Manns, B. J. (2009). Using effectiveness and costeffectiveness to make drug coverage decisions: a comparison of Britain, Australia, and Canada. JAMA 302, 1437-1443.

Docteur, E., and Paris, V. (2008). Improving Health System Efficiency: Achieving Better Value for Money. Brussels: Joint European Commission/OECD Conference.

Dubois, D. J. (2010). Grand challenges in pharmacoeconomics and health outcomes. Front. Pharmacol. 1:7. doi:10.3389/fphar.2010.00007

European Commission. (1989). Council Directive 89/105/EEC of 21 December 1988 Relating to the Transparency of Measures Regulating the Prices of Medicinal Products for Human Use and their Inclusion in the Scope of National Health Insurance Systems, European Commission: Brussels, 8-11.

European Commission. (2005). Pharmaceutical Forum. Available at: http://ec.europa.eu/pharmaforum/

European Medicines Agency. (2009). Report on Workshop on the Development of an EMEA Transparency Policy. Available at: http://www.ema. europa.eu/docs/en_GB/document_ library/Report/2009/10/WC500005 270.pdf

International Society for Pharmacoeconomics and Outcomes Research (ISPOR). (2011). ISPOR Tools for Health Care Decision Makers. Available at: http://www.ispor.org/work paper/UseOfResearch_index.asp

Kristensen, F. B., Lampe, K., Chase, D. L., Lee-Robin, S. H., Wild, C., Moharra, M., Garrido, M. V., Nielsen, C. P., Røttingen, J. A., Neikter, S. A., Bistrup, M. L., and The European Network for Health Technology Assessment. (2009). Practical tools and methods for health technology assessment in Europe: structures, methodologies, and tools

other reason for that matter, such as differences in the objectives and priorities of the different national health care systems.

Future discussions between Member States should strive at defining and triggering such a dual system with an integrated approach for relative effectiveness and EU medical need assessment and a coordinated/exchange based approach for value for money assessment. Table 1 illustrates the desired shift as compared to the current situation.

developed by the European network for health technology assessment. Int. J. Technol. Assess. Health Care 25, $1-8$.

Lampe, K., Mäkelä, M., Garrido, M. V., Anttila, H., Autti-Rämö, I., Hicks, N. J., Hofmann, B., Koivisto, J., Kunz, R., Kärki, P., Malmivaara, A., Meiesaar, K., ReimanMöttönen, P., Norderhaug, I., Pasternack, I., Ruano-Ravina, A., Räsänen, P., Saalasti-Koskinen, U., Saarni, S. I., Walin, L., Kristensen, F. B., and The European Network for Health Technology Assessment. (2009). The HTA core model: a novel method for producing and reporting health technology assessments. Int. J. Technol. Assess. Health Care 25, 9-20.

National Institute for Health and Clinical Excellence. (2005). NICE Citizens Council Report Ultra Orphan Drugs. Available at: http://www.nice.org. uk/niceMedia/pdf/boardmeeting/ brdjan05item4.pdf

National Institute for Health and Clinical Excellence. (2007). Final Appraisal Determination - Bortezomib Monotherapy for Relapsed Multiple Myeloma. Available at: http://www.nice.org.uk/guidance/ index.jsp?action $=$ folder $\& \mathrm{r}=$ true $\&$ $\mathrm{o}=35152$

O’Donnell, J. C., Pham, S. V., Pashos, C. L., Miller, D. W., and Smith, M. D. (2009). Health technology assessment: lessons learned from around the world - an overview. Value Health 12(Suppl. 2), S1-S5.

Raftery, J. (2006). Review of NICE's recommendations, 1999-2005. BMJ 332, 1266-1268.

Raftery, J. (2010). Multiple sclerosis risk sharing scheme: a costly failure. BMJ 340, c1672.

Rawlins, M. D., and Culyer, A. J. (2004). National Institute for Clinical Excellence and its value judgments. $B M J$ 329, 224-227.

Sorenson, C., Drummond, M., and Kanavos, P. (2008). Ensuring Value for Money in Health Care: The Role of HTA in the European Union. Copenhagen: European Observatory on Health Systems and Policies.

Sudlow, C., and Counsell, C. (2003). Problems with the UK Government's risk sharing scheme for assessing drugs for multiple sclerosis. BMJ 326, 383-392.

Tilson, L., and Barry, M. (2010). Recent developments in 
pharmacoeconomic evaluation in Ireland. Expert Rev. Pharmacoecon. Outcomes Res. 10, 221-224.

Van Wilder, P., and Dupont, A. (2008). Introducing evidencebased medicine in reimbursement procedures: does it affect the outcome? Value Health 11, 784-787.

Webb, D. (2009). Early Assessment of Cost-Effectiveness. Edinburgh:
Congress of the European Association for Clinical Pharmacology and Therapeutics.

Conflict of Interest Statement: Financial support for this research project was received from RIZIV/INAMI, the Belgian third-party payer. The authors have no conflicts of interest that are relevant to the content of this manuscript.
Received: 28 June 2011; accepted: 18 September 2011; published online: 11 October 2011.

Citation: Annemans L, Cleemput I, Hulstaert F and Simoens S (2011) Valorising and creating access to innovative medicines in the European Union. Front. Pharmacol. 2:57. doi: 10.3389/fphar.2011.00057

This article was submitted to Frontiers in Pharmaceutical Medicine and Outcomes
Research, a specialty of Frontiers in Pharmacology.

Copyright () 2011 Annemans, Cleemput, Hulstaert and Simoens. This is an openaccess article subject to a non-exclusive license between the authors and Frontiers Media SA, which permits use, distribution and reproduction in other forums, provided the original authors and source are credited and other Frontiers conditions are complied with. 